\title{
Pulmonary infection with Nocardia caviae in a patient with diabetes mellitus and liver cirrhosis
}

\author{
B SAlH, C FEgAN, A HUSSAIN, A JAUlim, K WhALE, A WEBb \\ From Monsall Hospital, Manchester
}

\begin{abstract}
The first reported case of pulmonary infection with Nocardia caviae in Britain occurred in a 67 year old woman, in whom diabetes mellitus and liver cirrhosis probably contributed to impaired cell mediated immunity. She was successfully treated with gentamicin $60 \mathrm{mg}$ eight hourly and sulphadimidine $500 \mathrm{mg}$ six hourly for six months and then with sulphadimidine $2 \mathrm{~g}$ /day for a further three months. When Nocardia caviae is isolated from the sputum of a patient with chronic respiratory infection and fever, effective treatment should be started promptly.
\end{abstract}

Nocardia organisms are Gram positive, partially acid fast, branching actinomycetes which uncommonly cause illness in man. Of a total of 17 cases of nocardiosis reported over the last three years in the United Kingdom $(1984,1985$, and 1986), 12 were due to Nocardia asteroides and the remainder due to untyped Nocardia species (Public Health Laboratory Service, unpublished). We report the first case in the UK of a patient with infection due to Nocardia caviae, an organism first isolated from an infected ear of a guinea pig by Snijders in $1924 .^{1}$

\section{Case report}

The patient is a 65 year old Pakistani woman with diabetes mellitus, who as a strict Muslim neither drinks alcohol nor smokes cigarettes; she has been resident in Britain for 12 years. She gave a two year history of intermittent fever, cough productive of persistently green sputum, malaise, and weight loss. Numerous sputum cultures carried out at the referring hospital had been negative for Mycobacterium tuberculosis and other mycobacteria, and the chest radiograph had shown patchy consolidation in both lung fields, with relative sparing of the apices (figure). She had had antituberculosis chemotherapy for almost one year and oral corticosteroids empirically for two to three months, without any clinical or radiological improvement. Examination after referral showed an obviously unwell, febrile, emaciated woman. She was breathless and had sinus tachycardia and crackles at the left base. She had abdominal swelling due to ascites but no apparent hepatosplenomegaly. Neurological examination at this stage showed nothing abnormal.

Investigations showed: haemoglobin $11.2 \mathrm{~g} / \mathrm{dl}$, white cells

Address for reprint requests: Dr B S Salh, Department of Chest Diseases, Monsall Hospital, Manchester M10 13WR.
$14.8 \times 10^{9} / 1(80 \%$ neutrophils), erythrocyte sedimentation rate $66 \mathrm{~mm}$ in one hour, and normal urea and electrolyte concentrations apart from sodium $127 \mathrm{mmol} / 1$. No malarial parasites were seen on the blood film. Liver function was deranged: serum bilirubin 50 (normal 1-20) $\mu \mathrm{mol} / 1$, alkaline phosphatase 1050 (35-105) IU/1 (almost all hepatic in origin), alanine aminotransferase 77 (0-35) IU/1, albumin 13 (33-48) $\mathrm{g} / \mathrm{l}$, total protein $63(60-80) \mathrm{g} / \mathrm{l})$. The prothrombin ratio was 1.8. No markers for hepatitis B infection were detected. Serum amylase activity was 115 (70-300) IU/1. Initial blood and stool cultures showed nothing remarkable. Abdominal ultrasound examination indicated that the liver was of abnormal texture and free fluid was present in the peritoneal cavity. An ascitic tap showed the fluid to be a transudate. A review of the histological appearances of the previous liver biopsy specimen showed well advanced micronodular cirrhosis.

The first indication that the patient had a nocardial infection was the finding of fine Gram positive branching filaments on a Gram stain of sputum. After five days of culture two colony types were identified, one white and the other off white, which were confirmed as Nocardia caviae by the Mycological Reference Laboratory of the Central Public Health Laboratory. Antibiotic sensitivity testing was performed by a disc diffusion method, with DST agar (Oxoid Ltd) enriched with $5 \%$ lysed horse blood, the Oxford strain of Staphylococcus aureus being used as the control organism. The organism was sensitive to gentamicin and sulphadimidine but resistant to trimethoprim, rifampicin, and all the cephalosporins tested. The patient was therefore given intravenous gentamicin $60 \mathrm{mg}$ eight hourly and oral sulphadimidine $500 \mathrm{mg}$ six hourly. Within one week of her starting treatment the pyrexia had subsided, the white cell count had decreased to $9 \cdot 2 \times 10^{9} / 1$ and the patient felt somewhat better. Sputum cultures for Nocardia caviae were repeatedly positive for two months, though the organism ceased to appear in the green stained sputum within three weeks of the start of effective treatment. During her recovery three main problems were encountered: firstly, unsteadiness of gait, which computed tomography showed to be due to a left cortical infarct, and from which she made a reasonable recovery; secondly, hypocalcaemic tetany (serum calcium $1.69 \mathrm{mmol} / \mathrm{l}$ ), which was resistant to calcium supplements; and, thirdly, severe paraesthesiae affecting the hands and feet. The serum magnesium concentration was $0.3 \mathrm{mmol} / 1$ and when it was restored to normal with magnesium gluconate supplements both the tetany and the paraesthesiae disappeared. Mild impairment of control of diabetes mellitus warranted temporary insulin treatment before she reverted to oral hypoglycaemic drugs.

The patient was treated with gentamicin and sulphadi- 


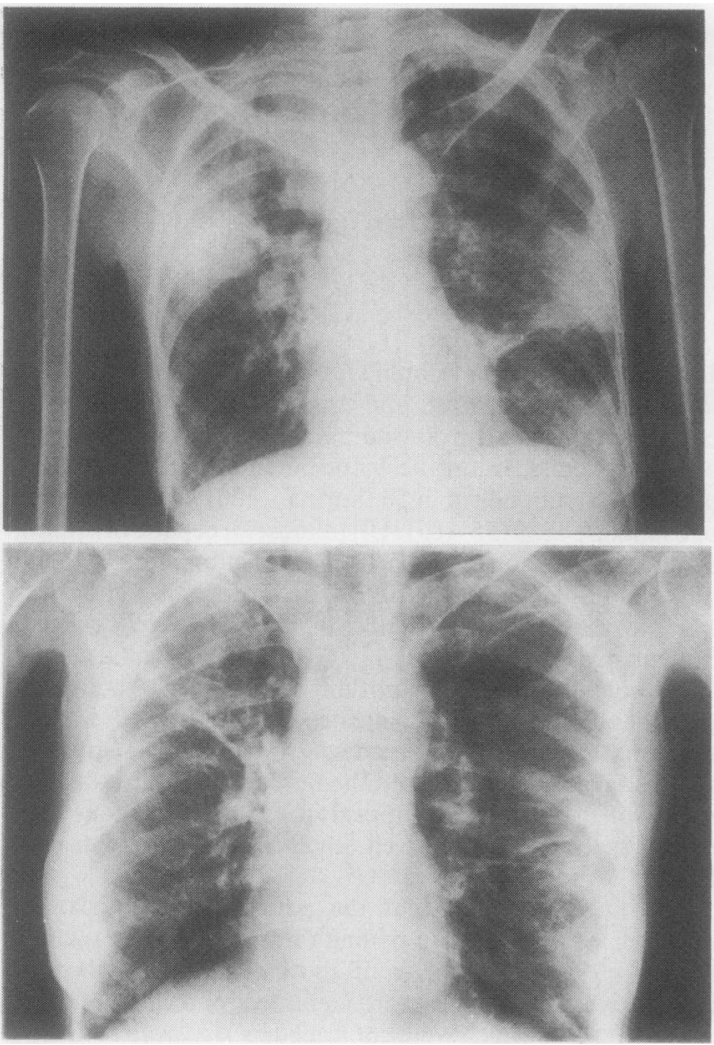

Chest radiograph $(A)$ on admission, showing patchy consolidation in both lung fields, and (B) after six months, showing substantial resolution of the consolidation.

midine for six months ${ }^{2}$ and then with sulphadimidine $2 \mathrm{~g} /$ day for a further three. Nocardia organisms were grown from her sputum two months after the start of treatment. The ascites resolved with treatment with spironolactone. A repeat chest radiograph six months later (figure, B) shows substantial resolution of the patchy consolidation.

Both the serum magnesium and the serum calcium concentrations have remained normal since the patient stopped gentamicin treatment.

\section{Discussion}

This is the first reported case of pulmonary infection with Nocardia caviae in Britain. As is often the case, it occurred in an immunocompromised setting, both diabetes mellitus and liver cirrhosis probably contributing to impaired cell mediated immunity. ${ }^{34}$ Disseminated infection due to this organism is invariably fatal. ${ }^{5}$

The isolation of Nocardia caviae from the sputum in a clinical setting of chronic respiratory infection and pyrexia is highly significant, and should prompt effective treatment.
Occasionally, however, the organism occurs as an airborne contaminant, and occasionally it is isolated from sputum in the absence of any overt pulmonary disease.

Sulphonamides are the drugs of choice for treatment of all forms of nocardiosis. Trimethoprim is reported to act $\mathbb{\varnothing}$ synergistically with these agents ${ }^{6}$ but this has not yet been clearly established. In this particular case antibiotic sen- $\infty$ sitivity testing indicated that the organism was resistant to $\overrightarrow{0}$ trimethoprim but sensitive to gentamicin, so gentamicin was used in combination with sulphadimidine.

The metabolic complications seen in this patient are presumed to be secondary to gentamicin induced renal $\vec{x}_{x}^{\vec{x}}$ magnesium loss, previously reported with total doses exceeding $10 \mathrm{grams}^{7}$ Our patient had been given a total dose of $21 \mathrm{~g}$. by the time hypomagnesaemia and hypocalcaemia were $\overrightarrow{-}$ observed. Impaired parathyroid hormone secretion is thought to provide a mechanism for this metabolic distur- $-\omega$ bance, but peripheral resistance to the action of this hormone음 also plays a part. $^{8}$ Both serum magnesium and serum calcium concentrations have remained normal in this patient after she $Z$ stopped the gentamicin.

Unfortunately there are no characteristic radiological changes associated with pulmonary nocardiosis, which can appear as consolidation, nodules (often cavitating), inter- $\mathbb{D}$ stitial changes, pleural effusion, and lymphadenopathy. ${ }^{9}$

In summary, this case illustrates that direct Gram staining of sputum may provide a clue to the aetiology of chronic pulmonary infection where tuberculosis appears to have been ruled out. Organisms such as actinomycetes are also likely to be missed by routine culture methods owing to their slow growth, as cultures are usually not continued for longer than 48 hours and anaerobic cultures are not usually performed unless there is a specific clinical indication.

\section{References}

1 Snijders EP. Verslag van het wetenschappenllijk gedeelte der vergaderingen van der afdelling Sumatra's oostkurst. Geneesked Tijdr Nederlandisch Indies 1924;64:75.

2 Weed LA, Anderson HA, Good CA, Reggenstoss AH. Nocardiosis: clinical, bacteriologic and pathologic aspects. $N$ Engl $J_{\sigma}^{\times}$ Med 1955;253:1137-43.

3 Palmer DL, Harvey RJ, Wheeler JK. Diagnostic and therapeutic considerations in Nocardia asteroides infection. Medicine (Bal- $\mathrm{O}$ timore) 1974;53:391-401.

4 Thomas HC. The role of the liver in immunological disease. Proc $R_{0}$ Soc Med 1977;70:521-5.

5 Arroyo JC, Nichols S, Carroll GF. Disseminated Nocardia caviae $\frac{D}{O}$ infection. Am J Med 1977;62:409-12.

6 Wallace RJ Jr, Septimus EJ, Williams TW Jr, Conklin RH, Satterwhite TK, Bushey MJ, Hollowell DC. Use of trimeth- - S oprim-sulphamethoxazole for treatment of infections due to N Nocardia. Rev Infect Dis 1982;4:315-25.

7 Bar RS, Wilson HE, Mazzaferri EL. Hypomagnesemic hypocal- $\mathbb{W}$ caemia secondary to renal magnesium wasting. Ann Intern Med 1975;82:646.

8 Rude RK, Oldham SB, Sharp CF, Singer FR. Parathyroid hormone secretion in magnesium deficiency. J Clin Endocrinol $\mathbb{\mathscr { D }}$ Metab 1976;42:707-10.

9 Feigin DS. Nocardiosis of the lung: chest radiographic findings in 7 21 cases. Radiology 1986;159:9. 